\title{
Manifeste pour une évolution industrielle
}

\section{Commitment to an industrial evolution}

\author{
Theodor Felezeu ${ }^{1}$ \\ ${ }^{1}$ ISEP - Ecole d'ingénieurs du Numérique, Paris, France, theodor.felezeu@isep.fr
}

RÉSUMÉ. L'industrie a le devoir d'un renouveau sans précédent pour répondre aux défis proposés par un contexte actuel complexe et particulièrement critique du point de vue social, économique ou environnemental. L'article se propose d'analyser les leviers que l'industrie peut activer en vue de s'inscrire dans une innovation pérenne, également contributrice à une réappropriation, par l'Homme, du sens de son évolution.

ABSTRACT. There is a need for a "new industrial deal » at a critical time in History, regarding the social, economic or environmental situations we go through. Article's goal is to perform an analysis of the main principles for the industries to follow, in order to stimulate sustainable innovation and play an active role in mankind's evolution.

MOTS-CLÉS. Industrie, innovation, emplois, économie, environnement, stratégies, technologies.

KEYWORDS. Industry, innovation, employment, economy, environment, strategies, technologies.

Si le terme de « révolution industrielle » résonne à nos oreilles (nous en serions déjà à l'orée de la quatrième ${ }^{1}$, et ce depuis une première allusion aux « usines intelligentes » lors du salon CEBiT à Hanovre en 2010 (CEBIT, 2016)), la notion « d'évolution » tend elle, à nous rendre plus perplexe.

A cela, trois causes majeures possibles : en premier lieu, les évolutions sont des perles rares. Selon Ken Wilber repris par Frédéric Laloux, nous serions en train de vivre une cinquième transformation majeure dans l'histoire de l'humanité, à mettre en perspective de quatre révolutions industrielles sur une durée d'à peine deux siècles (LALOUX, 2014). Ainsi, après avoir successivement écumé les ères magiques/ tribales, traditionnelles/agricoles, scientifiques/industrielles et postmodernes/informatiques, nous serions en cours d'expérimentation d'une profonde mutation holistique. Jean Staune explicite ainsi cinq révolutions associées à cette mutation (STAUNE, 2015), (STAUNE, 2016) : technologique, conceptuelle, économique, sociétale et managériale. En partant de ces postulats, nous pourrions ainsi nous interroger de quelle façon l'industrie (s')intègre (à) cette vision d'ensemble, dont les tenants et les aboutissants ne se réduisent plus au seul cadre productiviste à proprement parler.

La seconde cause de perplexité à l'égard de la notion d' "évolution 》 réside dans l'extrême complexité que sous-entend ce terme au sein de la société actuelle, aux impacts multiples et fabuleusement imbriqués, au premier rang desquels l'emploi et l'environnement, qui en constituent désormais ses clés de voute. Là encore, quelles peuvent être les réponses apportées par un renouveau industriel à ces défis politiques majeurs?

Enfin, alors que nous avons une certaine affinité avec les révolutions, que ce soit pour renverser des pouvoirs existants ou pour commercialiser l'Iphone dernier cri, nombreux sont ceux qui, lorsqu'on ose aborder l'évolution, s'empressent d'invoquer le Darwinisme. Sans forcément tomber dans cet aspect réductionniste, voire, à l'opposé, redéfinir la notion elle-même, nous avons (et a minima le monde industriel a) le devoir de lui redonner un sens dans le contexte actuel ; de la traiter avec toute l'exigence que requiert la complexité du monde dans lequel nous vivons aujourd'hui, et dans celui dans lequel nos enfants et petits-enfants évolueront demain. Ainsi, l'on pourra se demander de quelles manières aborder les inventions et innovations industrielles en vue d'offrir à cette exigence collective (la nôtre), une dynamique pérenne, soutenable.

1. On peut distinguer les 4 révolutions comme suit : 1ère révolution (XIX $\mathrm{X}^{\mathrm{e}}$ siècle) : machines à force hydraulique (vapeur) / 2ème (début $\mathrm{XX}^{\mathrm{e}}$ ) : énergie électrique et production de masse (Taylorisme), $3^{\mathrm{e}}$ (années 1970) : électronique et informatique (Toyotisme), $4^{\mathrm{e}}(2010)$ : internet des objets, Big Data, fabrication modulaire et additive. 
Ainsi, après avoir dressé les constats et mis en avant les risques encourus par les politiques industrielles actuelles, l'article explorera trois lignes directrices sur lesquelles l'industrie doit se baser pour se réinventer en vue de s'inscrire sur une trajectoire réellement durable: l'industrie X.0 ; les valeurs au cœur des systèmes de production ; l'éclosion de nouveaux modèles économiques. La fin de l'article sera constituée d'un éclairage sous forme de corolaires et d'une synthèse consacrés aux impacts de cette évolution industrielle sur les cultures, les visions, stratégies, processus et outils de l'innovation.

\section{Constats, sentiments partagés et risques encourus}

En vue de saisir certains phénomènes globaux au travers du prisme industriel, intéressons-nous à quelques données relatives aux effets économiques, sociaux et environnementaux engendrés par les politiques industrielles menées à ce jour :

- la part de l'industrie dans l'économie mondiale s'élevait, en 2014, à 27,6\% (BANQUE-MONDIALE, 2016);

- 3,1 millions de salariés y travaillaient en France en 2014, hors intérim (GOUVERNEMENT, 2016) ;

- l'avènement de l'industrie 4.0 impliquerait la destruction de 5 millions d'emplois (LATRIBUNE.fr, 2016);

$-1 / 2000$ serait le rendement terres rares / déchets toxiques (KAIMAN, 2014) intervenant dans l'exploitation, à date, des 17 métaux dits « stratégiques » utilisés notamment dans la production d'écrans tactiles, de lentilles de contact, d'éoliennes, d'ampoules basse consommation ou bien encore de batteries de voitures ;

- 1,36 / 2,25 / 1,18 soit les coefficients multiplicateurs des concentrations de GES (gaz à effets de serre) depuis 1850 pour le CO2 / CH4 / N2O et étant, selon le GIEC (Groupe d'Experts Intergouvernemental sur l'Evolution du Climat) à l'origine d'un réchauffement climatique désormais avéré (GIEC, 2014).

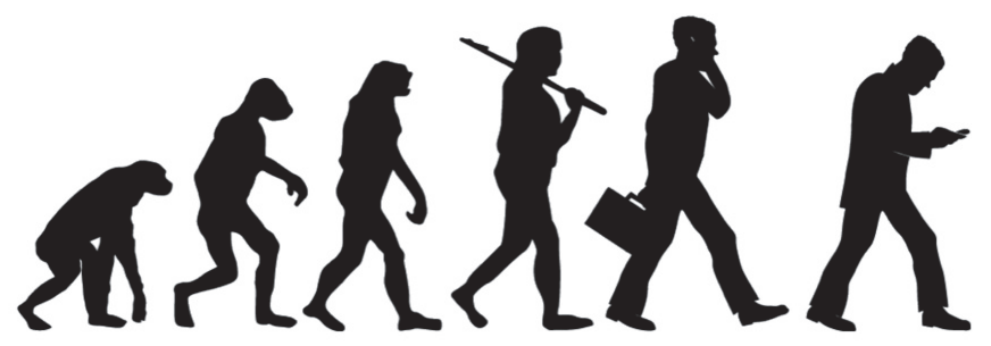

Figure 1.1. Homo Numeris

Dans l'état actuel des choses, il paraît difficile de se réjouir de ces constats ainsi que des perspectives induites, et ce dans le cas où des mesures tangibles, impactant différentes échelles spatio (sociétés, territoires, nations / puissances économiques, consommateurs)-temporelles (très court et moyen-terme) ne seraient pas entreprises avec une exigence suffisante - i.e. permettant d'harmoniser de façon pérenne les Hommes et leur(s) environnement(s).

Ainsi, si nous manquons notre rendez-vous avec ces défis stratégiques que représentent les suffixes 《 -nomie » et «-logie » de l' " éco » (divers intellectuels tels que Jacques Attali, Christian Saint-Etienne ou encore Philippe Baumard (BAUMARD, 2012), (BAUMARD, 2014) ayant souligné une prépondérance de l'action tactique et du court-termisme au détriment de la prise en considération des impacts sur le long terme), il encourt un fort risque de "Game Over », que nos sociétés continuent à entretenir et même à conforter en forgeant l'épée de Damoclès qui accompagne d'ores et déjà le futur de nos enfants et petitsenfants... 
...à moins que nous soyons encore à même de continuer dans une logique mythologique de sacrifice, entretenue (consciemment ou non) par un modèle économique dominant profondément inégalitaire visà-vis non seulement des générations actuelles (en 2016, 1\% des habitants de la planète possède plus que les $99 \%$ autres (OXFAM, 2015)), mais également de celles futures (accélération progressive de l'économie de la dette depuis les années 70 (INSEE, 2016)).

En d'autres termes, à chaque fois que les décisions se fondent sur la recherche de la rentabilité maximale, entraînant le plus souvent dans son sillage logiques de compétitivité débridée, de croissance aveugle (un mythe de plus en plus maltraité au vu du paradigme actuel liant démographie - et donc pratiques de consommation - et production - à date, notamment, pourvoyeuse de déchets), un risque substantiel reléguant des vies et des trajectoires humaines au second plan, existe. Les conséquences de ce risque sont pointés du doigt par quelques singletons de la sphère intellectuelle tel que le philosophe Jean-François Mattéi dans son dernier ouvrage (MATTEI, 2014) ou le juriste Alain Soupiot, professeur au collège de France (SUPIOT, 2015).

Pour exemple, la soustraction de capitaux des appareils de production (eu égard aux enjeux d'utilité sociale et environnementale de ces derniers) de façon disproportionnée (grâce à l'outil (hyper)-financier), via un paradigme d'actionnariat fondé sur la maximisation du profit, qui a pris naissance dans les années 70 pour s'épanouir à partir du milieu des années 80 (voir figure ci-dessous).

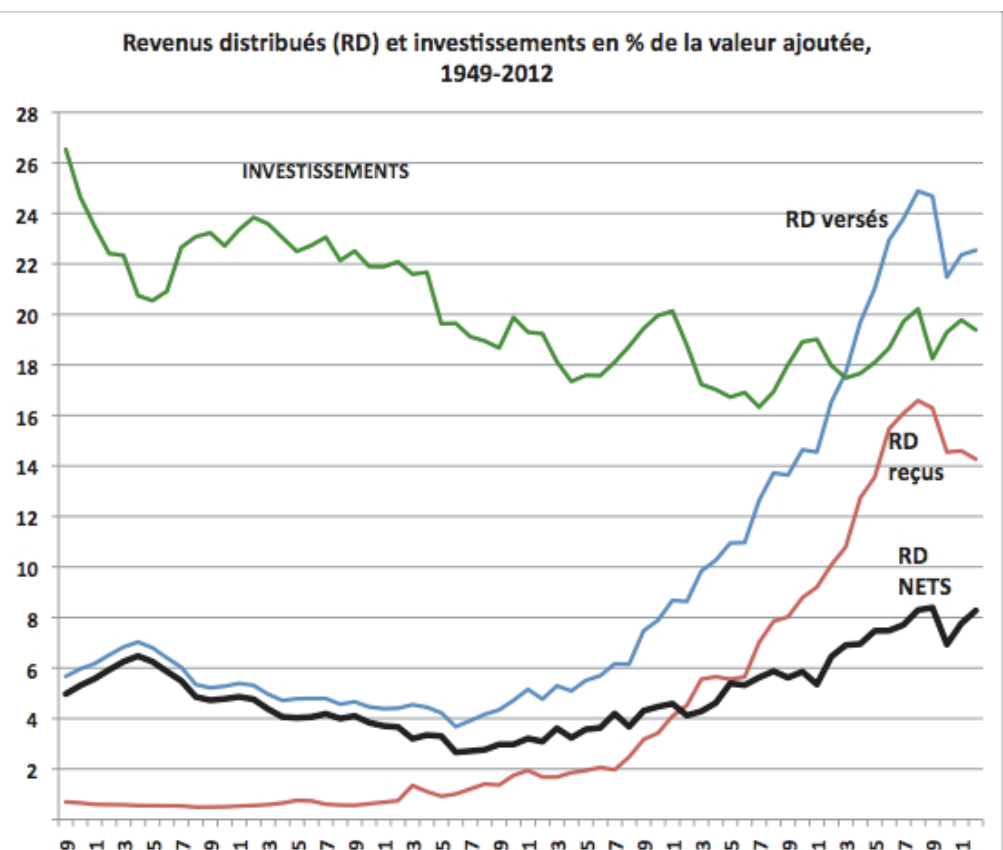

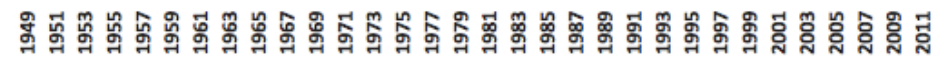

Figure 1.2. Revenus distribués aux actionnaires des sociétés non financières (SNF) en France en comparaison des investissements réalisés (GADREY, 2014) - Source : Jean Gadrey

Jean Gadrey, professeur honoraire d'économie à l'Université de Lille 1 nous éclaire : «En effet, on peut estimer qu'avec 3 à $4 \%$ de la valeur ajoutée dans les années 1960 à 1980, avant la révolution de la financiarisation, les dividendes et autres revenus distribués nets étaient alors compatibles avec un fonctionnement productivement efficace des entreprises. De sorte qu'avec 7,5 à $8 \%$ ces dernières années, on est autour de $4 \%$ de rente actionnariale improductive. [...] 40 milliards [...]. Pas seulement improductive d'ailleurs : destructrice aussi, car l'essentiel part dans des spéculations déstabilisatrices. » Imaginons ainsi l'effet d'une répartition de ces 10 à 15 milliards dans une industrie modernisée, aux moyens de production propres et aux équipes formées à affronter les défis de demain. 
Alors concrètement, quels choix s'offrent à nous ? Considérons quatre variantes :

- l'immobilisme, l'aveuglement - ou la loyauté (DENIS, 2014) : le monde avance selon une trajectoire immuable, sur la base de paradigmes et d'habitudes issus de l' " ancien monde » (croissance infinie, entretien des inégalités) avec en trame de fond des rafistolages tactiques à grand renforts de communication et d'un contrôle (somme toute illusoire à l'heure de l'internet) de l'opinion publique ;

- la fuite : oui, mais vers quoi ? En avant ? (cf. point précédent)

- la révolte : chère aux velléitaires-contestataires-révolutionnaires, comprend une déconstruction (souvent) violente des modèles passés, exaltant instincts primaires et replis identitaires, conflits et clivages civilisationnels, entraînant un risque de réaction en chaîne de pertes de repères et des coupures sociales, sociétales et transgénérationnelles ;

- la pertinence : fondée sur un couple prise de conscience / passage à l'action, expérimentée par des élites citoyennes créatives et engagées remettant en cause les paradigmes inadaptés du passé, progressant en cercles concentriques (du local vers le global) pour construire de façon performante et pérenne le bien-être des sociétés (humaines et environnementales) aujourd'hui et demain. Ce qui reviendrait (toutes proportions gardées) à transformer le bon vieux projet et son cycle en $\mathrm{V}$ (étude, spécification, conception, validation, livraison) par un modèle agile «Lean » (prototypage, test / analyse, implémentation) parfaitement décrit dans (RIES, 2011).

Présenté comme ceci, il suffit de décrypter l'actualité pour avoir un aperçu de l'agitation ambiante dont les signaux (de moins en moins faibles) apparaissent au travers des crises économiques, environnementales, politiques, religieuses ou identitaires que nous vivons. En fonction des influences exercées ou subies, nous sommes amenés à nous positionner vis-à-vis de ces quatre choix, ce que nous faisons de façon quasi permanente, concomitante et a fortiori chaotique, prisonniers de flots (et pas uniquement migratoires ou financiers) qui apparaissent de plus en plus difficiles à contrôler. Ce qui n'est pas sans rappeler les agitations éprouvées par nos ancêtres dans des circonstances contextuellement liées à des retournements de régimes politiques, à des bouleversements technologiques ou encore à des catastrophes naturelles, signe là encore d'une profonde mutation. Le "charme» de notre époque réside dans le fait que tous ces changements interfèrent à une échelle planétaire et qu'il nous est donné l'opportunité (ou le risque) de les expérimenter de notre vivant.

Le premier problème associé à cette situation inhabituelle, voire « sur-humaine » (elle l'est de facto par le biais des états et des entreprises, acteurs majeurs de ces enjeux), est que la quatrième voie paraît manifestement sous-représentée par rapport, non pas aux trois autres, mais à l'effet induit dans le temps par les trois autres : l'impact de l'héritage laissé sur nous par 200 ans d'exploitations féroces, d'idéologies totalement antagonistes, de formes archaïques de conscience du progrès, d'éducation des masses, d'accentuation des logiques compétitives, de dérives et d'abandons divers, etc. Et finalement, de choix et de prises de positions jugées opportunes au moment de leur implémentation (un moment qui est dépassé, et qui continue, au fur et à mesure que nous avançons de l'être de plus en plus vite), qui finissent par s'ancrer dans des sillons idéologiques marqués et des habitudes, qui, lorsque vient le moment d'impulser le changement, sont si complexes à faire évoluer.

Le second problème réside dans la façon d'aborder cette quatrième voie, que beaucoup d'entre nous ne considérons pas avec suffisamment de globalité, de manière suffisamment holistique, enclavés dans de vieilles querelles de chapelle (beaucoup de chapelain-e-s en France notamment), érigeant d'incessants débats sur la façon de considérer l'économie (de marché vs solidaire), la société (entrepreneuriat vs assistanat), l'entreprise (mastodonte vs start-up), le numérique (plateformes vs blockchain), l'action politique (étatique/interventionniste vs citoyenne/libertarienne), etc. 


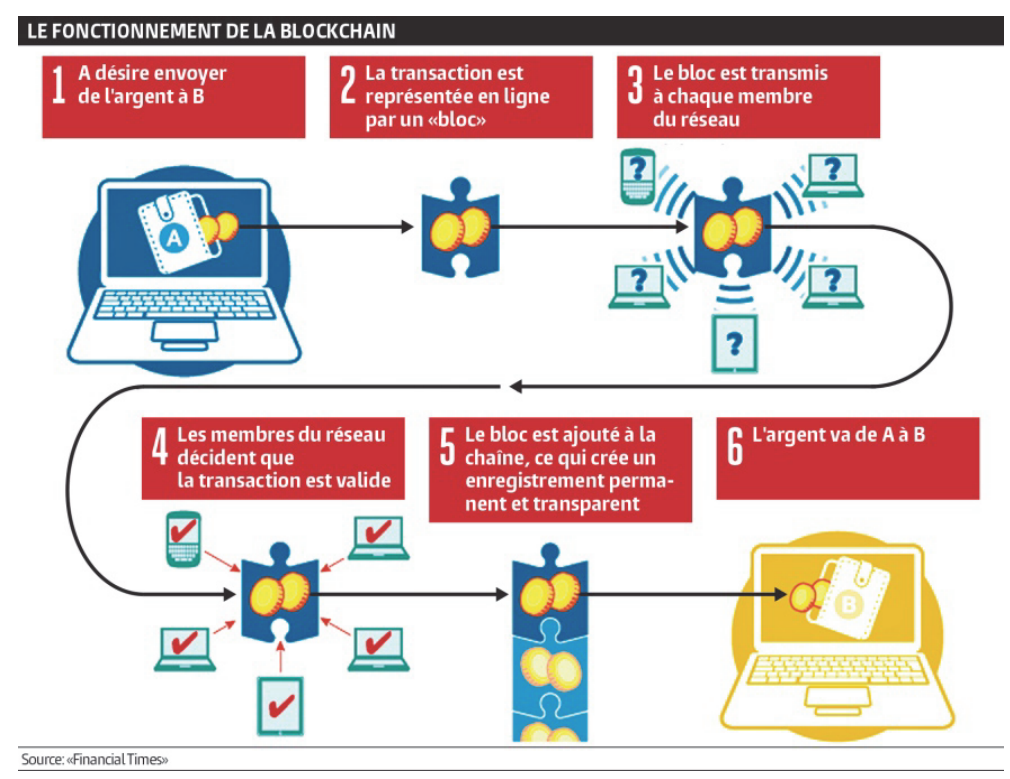

Figure 1.3. Principes de fonctionnement de la blockchain, un outil à explorer - Source : Financial Times

Comme si le cartésianisme ${ }^{2}$, proprofondément ancré dans les valeurs, avait eu raison de l'art du paradoxe qui s'avère pourtant utile à manier dans un univers pluriel, complexe... « quantique ».

De surcroît, lorsque cette voie est choisie et adressée avec de fort bonnes intentions, la plupart de celles-ci auraient été sans doute louées dans un contexte moins exigeant : moins polluer, infléchir la courbe du chômage, se contenter d'aménagements dans l'éducation alors que de vraies réformes structurelles s'imposent...

Mais au-delà de ressentir que cela ne suffit plus (sensation d'être pris en otage au travers des sentiments d'urgence qui s'accumulent, largement liés aux crises passées et/ou celles annoncées), les dettes économiques, environnementales, sociales ne cessent de s'accroître.

Tout est une question de curseur serions-nous tentés de dire de façon plus ou moins indulgente. Et nous aurions sans doute raison, oui. Mais est-ce le plus important? Rien n'est moins sûr. En effet, si un consensus de plus en plus large s'accorde sur le fait que des changements s'imposent - et se mettent d'ailleurs en place - à tous les niveaux (éducation, santé, fiscalité, protection environnementale, production / consommation) l'absence d'actions stratégiques d'envergure (planétaire) fait encore cruellement défaut. Et combien même un embryon de pensée émergerait, il y aurait de grandes chances pour qu'il soit phagocyté par des bien-pensances dominantes, souvent atomisées et par voie de conséquence, non coordonnées et / ou à l'avantage de cercles de privilégiés (multinationales / puissances / lobbies).

\section{Focus sur la nouvelle donne industrielle}

Et l'industrie dans tout cela? A notre sens, compte-tenu des enjeux évoqués, elle a un rôle fondamental à jouer dans sa capacité à se renouveler. Elle ne peut se contenter d'une simple révolution, et ce quel que soit le « bond en avant» technologique, opérationnel, managérial ou social \& environnemental au sens de l'actuelle RSE (Responsabilité Sociale des Entreprises). Ce serait évidemment une avancée " louable ». Mais elle resterait tout à fait négligeable en termes d'impacts par rapport à un réel « changement des règles du jeu », tel que le prône l'industriel Gunter Pauli, initiateur de la Blue Economy (PAULI, 2015). Sa vision : non pas « moins » polluer, mais tout simplement ne pas polluer du tout ! Viser une production ZERO DECHET.

2. Au travers de ses deux composantes principales (le déterminisme - i.e. le lien « mécanique » entre la cause et l'effet, et le réductionnisme - i.e. la décomposition systématique d'une problématique en sous-tâches, chacune d'elles étant elle-même redécoupée jusqu'à ce qu'elles soient réduites à des modules spécifiques répondant, chacun, à un problème simplifié) participant notamment à une "atomisation" des rôles et des fonctions, à des clivages de type "généralistes" / "spécialistes" ou, plus globalement, à une vision du monde aboutissant à des courants de pensée empreints de manichéisme et à vis-à-vis duquel certains "maîtres" de la réflexion complexe tels que Jean Staune ou Edgar Morin (MORIN, 2011) s'interposent. 
Voici que nous entrons dans le vif du sujet. Faisons le point: transposée aux constats précités, l'industrie peut-elle s'affranchir des règles économiques et politiques en vigueur ? Pas véritablement. De l'avènement de l'uberisation et des bouleversements technologiques déjà bien enclenchés ? Pas d'avantage, comment le pourrait-elle ? A-t-elle cependant le devoir, l'exigence, de renouveler sa nature profonde pour répondre aux enjeux du futur? Tout dépend du sens que l'on (politiques, économistes, capitaines d'industrie, société civile) souhaite donner à ce futur. Le sens prime sur le curseur. L'innovation oui, mais avec et pour l'Homme.

IMPORTANT. Et donc, pour résumer : la nécessité d'une réinvention collaborative, pluridisciplinaire, basée sur des valeurs fondamentales et inaliénables, accompagnant et alimentant l'expérimentation créatrice des entrepreneur-e-s.

Dans le concret, cela pourrait reposer sur trois chantiers ambitieux, qui, notons-le, ont le bon goût de coexister dans des sphères séparées (encore que !), mais qui sont loin de coopérer eu égard des exigences soulevées en préambule :

- une industrie X.0 : numérique, connectée, collaborative et quasi sur-mesure ;

- illuminée par les valeurs de la renaissance : humain, formation, connaissance ;

- favorisée par des alternatives économiques : repenser le revenu, la valeur-travail, le partage.

\subsection{Au-delà de l'industrie 4.0}

Des trois chantiers, celui relatif à l'industrie connectée et à ses nouvelles formes de production semble être le plus compréhensible, a priori. Divers acteurs de l'univers du conseil notamment nous éclairent sur ces démarches, qui sont issues de la rencontre entre le progrès technologique et les techniques de lean développées par le monde industriel durant les 30 glorieuses, visant une production plus performante et plus optimisée. Ainsi, Kurt Salmon nous parle des principes clé de l'Industrie 4.0 (SALMON, 2015), « constitu[ant] une vraie rupture sur trois grandes innovations technologiques : la robotique collaborative, l'autoadaptation des systèmes de production (grâce aux capteurs intelligents et connectés) et le recours à la réalité augmentée. » Ainsi, après l'Allemagne (2010), le Royaume-Uni (2011) ou l'Italie (2012), la France a lancé son propre programme baptisé « Usine du Futur » en 2013. Dans le cadre d'une feuille de route comprenant 34 plans pour la nouvelle France industrielle, il vise à moderniser les industries - la France accuserait un retard de 40 milliards d'euros d'investissements dans le parc industriel par rapport à son voisin allemand - en les rendant compétitives et pourvoyeuses d'emplois (GOUVERNEMENT, 2014).

ÉNERGIES RENOUVELABLES
LA VOITURE POUR TOUS CONSOMMANT
MOINS DE 2 LITRES AUX 100 KM
BORNES ÉLECTRIQUES DE RECHARGE
AUTONOMIE ET PUISSANCE DES BATTERIES
VÉHICULE AUTONOME
AVION ÉLECTRIQUE ET NOUVELLE
GÉNÉRATION D'AÉRONEFS
DIRIGEABLES ET DRONES CIVILS
LOGICIELS ET SYSTĖMES EMBARQUÉS
LE SATELLITE À PROPULSION ÉLECTRIQUE
TGV DU FUTUR
NAVIRES ÉCOLOGIQUES
TEXTILES TECHNIQUES ET INTELLIGENTS
INDUSTRIES DU BOIS
RECYCLAGE ET MATÉRIAUX VERTS
RÉNOVATION THERMIQUE DES BÂTIMENTS
RÉSEAUX ÉLECTRIQUES INTELLIGENTS
QUALITÉ DE L'EAU ET GESTION DE LA RARETÉ

\author{
CHIMIE VERTE ET BIOCARBURANTS \\ BIOTECHNOLOGIES MÉDICALES \\ SANTÉ NUMÉRIQUE \\ DISPOSITIFS MÉDICAUX ET NOUVEAUX \\ ÉQUIPEMENTS DE SANTÉ \\ PRODUITS INNOVANTS POUR UNE \\ ALIMENTATION SÛRE, SAINE ET DURABLE \\ BIG DATA \\ CLOUD COMPUTING \\ E-ÉDUCATION \\ SOUVERAINETÉ TÉLÉCOMS \\ NANOÉLECTRONIQUE \\ OBJETS CONNECTÉS \\ RÉALITÉ AUGMENTÉE \\ SERVICES SANS CONTACT \\ SUPERCALCULATEURS \\ ROBOTIQUE \\ CYBERSÉCURITÉ \\ USINE DU FUTUR
}

Figure 2.1.1. Les 34 plans pour la nouvelle France Industrielle 


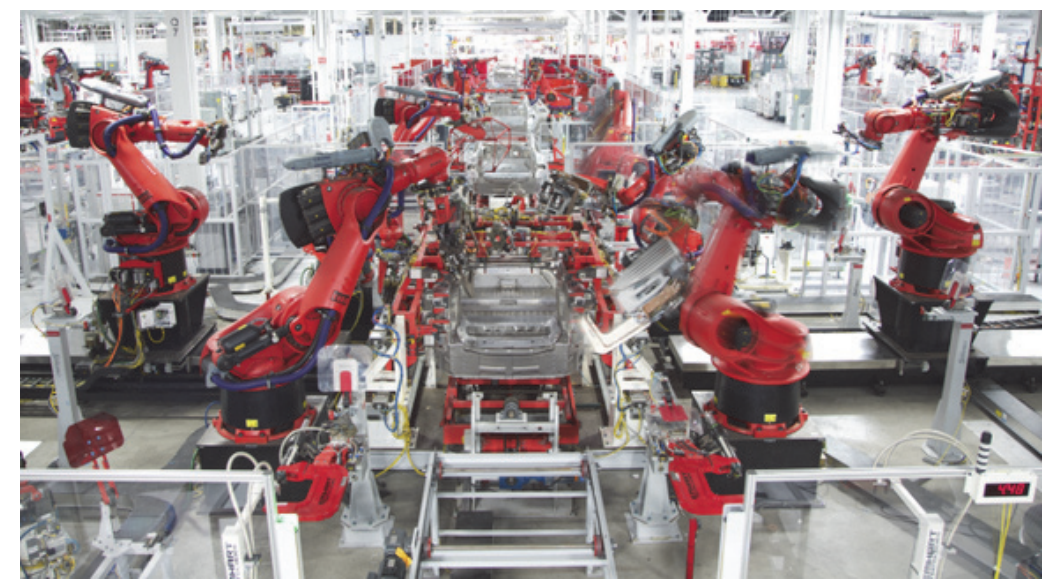

Figure 2.1.2. Usine TESLA - Fremont, USA. - Source : TESLA MOTORS

Le cabinet OPEO élargit quant à lui le débat (OPEO, 2014). Lors d'une présentation réalisée durant le rassemblement annuel de la BPI (Banque Publique d'Investissement - BPIFrance Inno Generation (BPI-FRANCE, 2016)) fin mai, qui regroupe chaque année l'ensemble des acteurs majeurs de la transformation numérique, grands groupes ainsi que des start-ups prometteuses, David Marchenaud et Michaël Valentin (ex-McKinsey) défendent le sujet de l'excellence opérationnelle. Levier pour rendre les entreprises agiles et économiquement performantes, l'avènement de l'industrie 4.0 redéfinit en profondeur les contours même de l'organisation dans l'entreprise (pilotage en unités agiles, communication et prises de décision horizontales, rôle clé du manager/coach) mais également à l'extérieur (transformation de la chaîne à valeur ajoutée avec le client qui s'invite dans l'unité de production). Pour OPEO, c'est dans la mise en cohérence des aspects techniques, organisationnels et humains que réside la clé de la réussite des usines du futur.

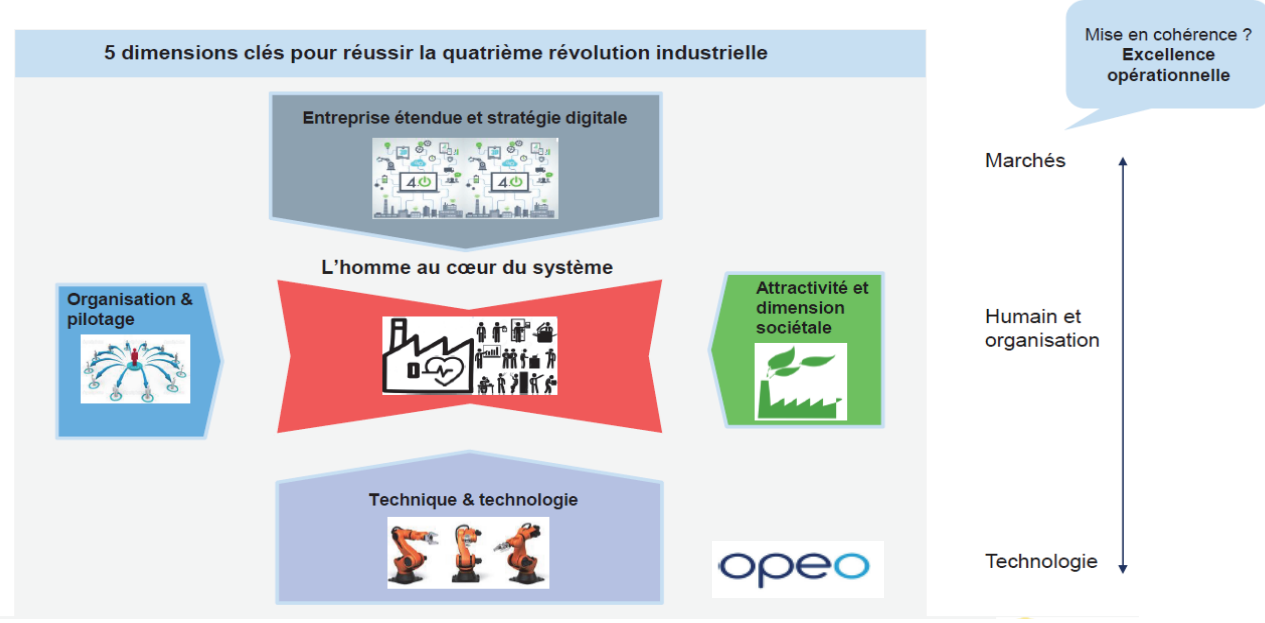

Figure 2.1.3. L'Homme au cœur de l'excellence opérationnelle - Source : OPEO

Ainsi, que ce soit au niveau Managérial ou Opérationnel, l'humain devra composer avec les quatre dimensions schématisées ci-dessus (OPEO, 2016) :

- l'appréhension des technologies et techniques de production (choix des outils les plus adaptés, formation et montée en compétences des équipes, relation homme-machine) ;

- les nouveaux modes d'organisation (délaissement progressif des structures pyramidales au profit d'unités autonomes de décision) ;

- la dimension attractive de l'entreprise (RSE favorisant l'embauche de profils de qualité, moins de turnover lié à des conditions de travail optimales) ;

- devant répondre à des marchés exigeants et imprévisibles (temps-réel, « client-roi ») via une entreprise orientée multi-canal (e-commerce, nouveaux circuits de distribution, marché mondialisé). 
Côté PATRIUM, initiative citoyenne dont l'objectif est l'accompagnement de PMI (Petites et Moyennes Industries) en difficulté, il est porté une vision dans laquelle la petite industrie dotée de moyens numériques est en capacité de reprendre sa place au cœur d'une économie uberisée, mettant directement en lien consommateurs et producteurs (PATRIUM, 2016).

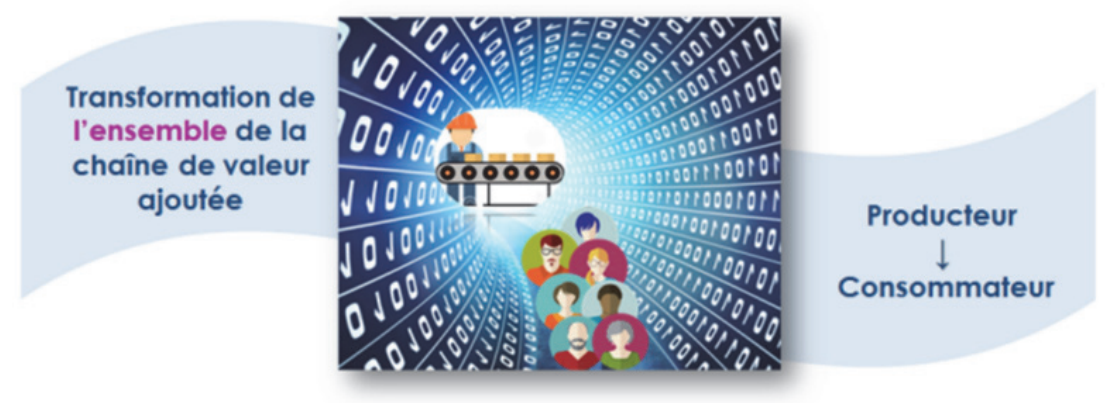

Figure 2.1.4. L'industrie uberisée

L'objectif est ici la sauvegarde des emplois et du patrimoine industriel potentiellement pérenne, sur les territoires, mis encore à mal par les délocalisations et les fermetures d'usines liées au manque de compétitivité associé à l'ère «pré-internet ». Grâce à une vision alliant numérique, travail collaboratif en réseaux de PMI et favorisant la production en petites quantités agiles à destination des marchés de traîne (des niches / tribus de consomm-acteurs fidèles et exigeants en termes de qualité, valeurs, principes, adressées à une échelle non plus simplement locale mais mondiale), Patrium porte l'espoir d'une réindustrialisation progressive, localement. Basé sur 25 ans d'expérience ayant permis la pérennisation d'une cinquantaine d'entreprises et plus de 1000 emplois dans des domaines aussi variés que la visserieboulonnerie, le textile, le bois ou la plasturgie, l'initiative s'est consolidée en 2014 via la création d'un Fonds d'Investissement Solidaire et Citoyen qui accompagne actuellement des entreprises à partir de sa région pilote, la Nouvelle-Aquitaine. L'idée sous-jacente forte est la transmission, aux générations futures, d'outils industriels pourvoyeurs d'emplois. Pour ce faire, l'aspect économique des financements se rapporte à la notion d'E3S (Economie Sociale, Solidaire et Solvable), dans laquelle le retour sur investissements autant social et sociétal que (très raisonnablement) financier. L'innovation réside ici dans la combinatoire d'une vision (la réindustrialisation possible), d'un modèle économique fondé sur le partage, basé sur des outils (numériques, collaboratifs, outil de production vendant aussi du service) avec une finalité de transmission (pérennité, emplois).

\section{Société Broussaud Textiles, Les Cars, Limousin \\ - Reprise en 2008 \\ $-300 \mathrm{~K} €$ investis \\ - 25 emplois pérennisés \\ $-25 \%$ de croissance en moyenne par an}

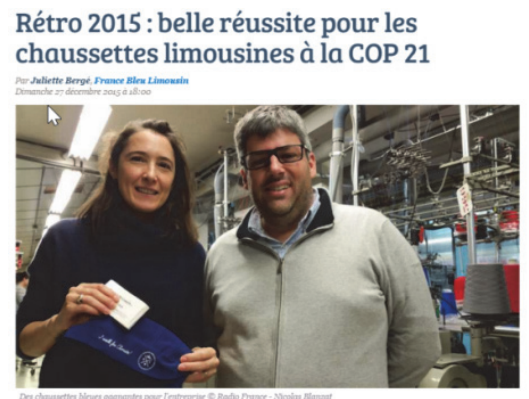

Encadré 2.1. Broussaud Textiles, pilote Patrium

Mention spéciale enfin pour le développement d'une industrie favorisant de plus en plus le traitement des déchets de façon performante et durable ainsi que la réparation et l'entretien des biens prolongeant leur durée de vie. Comme par exemple, la start-up SAVE, qui s'est positionnée sur la réparation de tout objet électronique dans un marché de 50 Milliards d'objets connectés à horizon 2020 et qui est passée en un an d'un chiffre d'affaires annuel de $100 \mathrm{~K} €$ à un chiffre d'affaires de 100K€ par jour (SAVE, 2016). 
Ainsi, l'innovation ne peut plus simplement se conjuguer avec la réponse à un besoin d'usage ou à une problématique systémique donnée. Elle doit aborder, fondamentalement, en conscience, les aspects sociaux et environnementaux contextualisés à notre époque. Sachant par exemple que $20 \mathrm{Kg}$ de matières premières servent à produire une puce de $0,09 \mathrm{~g}$ (ADEME, 2016), 50 Milliards de puces électroniques exigent l'utilisation de 10 Gigatonnes de matières premières (en équivalent « Harmony of the Seas ", cela représente près de 4500 paquebots). Le tout s'inscrivant dans une récurrence dictée par une fréquence de renouvellement des équipements à usage personnel de cinq à sept ans (en prenant en compte des hypothèses extrêmement raisonnables au niveau de la réparation et d'allongement des durées d'usage).

Cela signifie tout simplement que nous sommes amenés à puiser dans les réserves de la planète (non extensibles a fortiori) de manière récurrente, et grandissante. Le cas des terres rares est à lui seul l'archétype d'une exploitation nocive. Il risque bientôt d'être suivi, voire surpassé, par l'exploitation du lithium. Présagée par les 5 milliards de USD investis par l'entreprise Tesla Motors dans son projet de Giga-Factory dans le Nevada (TESLA, 2016), censé assurer à partir de 2017 la production des batteries lithium-ion pour l'ensemble du parc automobile de l'entreprise, soit un rythme de production estimé à 500000 véhicules par an. Et pour Tesla de prophétiser : « D'ici 2020, la Gigafactory atteindra sa capacité maximale et produira plus de batteries au lithium-ion par an qu'il n'en était produit dans le monde en 2013. » Inquiétant, compte tenu, de nouveau, des impacts afférents (PARASOTE, 2013).

« Celui qui croit qu'une croissance exponentielle peut continuer indéfiniment dans un monde fini est soit un fou, soit un économiste. » (Kenneth E. Boulding, cité dans Jump the Curve - Jack Uldrich, 2008)

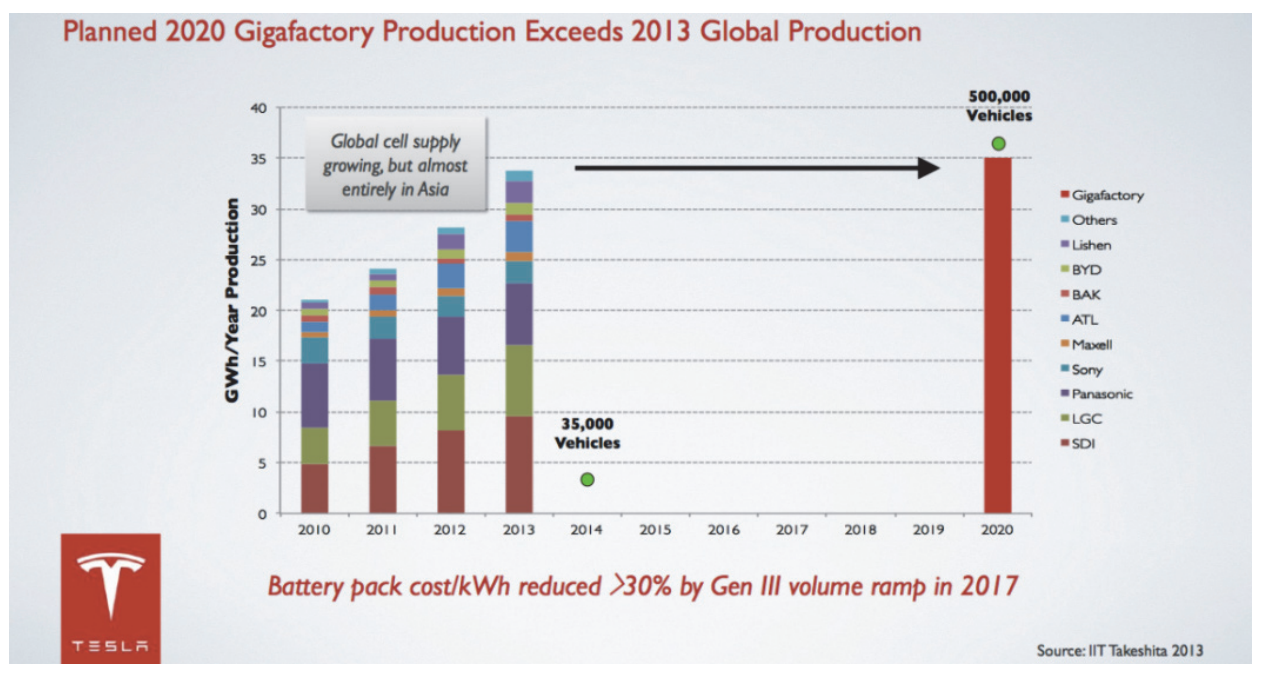

Figure 2.1.5. Tesla Gigafactory - Production planifiée de batteries Lithium-Ion - Source : IIT Takeshita 2013

Alors, à quoi peut donc bien servir une innovation aussi fabuleuse que la voiture électrique, censée moins polluer (son principal argument de vente), si, en parallèle, au cœur même de son processus de production, sont également engendrés des risques de dégradation de l'environnement ?

Combien même les processus de fabrication pourraient être améliorés dès aujourd'hui (et ils vont l'être de plus en plus) et les plus engageantes chartes Green signées (ce qui doit être encouragé), il apparaît complexe d'inverser la tendance actuelle : une augmentation progressive, massive, difficilement enrayable de la dette environnementale.

Dès lors, et ce afin de permettre à l'industrie X.0 de s'ancrer dans un sillon spatio-temporel réellement pérenne, comment ne pas évoquer la façon même de concevoir la production de biens et la façon d'en utiliser les services induits. Quel futur énergétique et écologique à l'industrie en face des besoins de 9,6 Milliards d'habitants à horizon 2050, c'est-à-dire demain matin? Il s'agit là de l'un des plus extraordinaires défis qu'il est donné à l'humanité de relever... 


\subsection{Pour une renaissance, mais sans pour autant mourir avant (ou alors sans trop souffrir)}

Il peut y avoir une tendance à croire que la souffrance (ou son attente) est pire que la mort. La mort restant à date, une inconnue, tandis que la souffrance, pouvant elle, être ressentie, communiquée, partagée. La plupart du temps, malheureusement, la mort s'accompagne de souffrances. Ainsi, la crise de 2008 aurait induit 500000 morts par cancer (LE-MONDE.fr, 2016), tandis que la pollution atmosphérique mettrait en danger nos cerveaux (FOUCART, 2014). Quant à la planète, l'exploitation du gaz de schiste entraînerait des tremblements de terre (BUSSARD, 2016) dans l'Oklahoma. Arrêtons-là le catastrophisme et agissons.

Changer les règles du jeu...

...cc'est mettre au cœur de de la « partie » industrielle trois ingrédients de premier plan :

- l'économie circulaire, permettant la réutilisation, le réemploi ou le recyclage (non pas de 99\%... mais) de $100 \%$ des ressources nécessaires à la fabrication du produit, du produit en lui-même et des déchets induits par les processus de fabrication ;

- l'économie de la connaissance, permettant le partage et la diffusion (infinies ?) des connaissances, savoir-faire et techniques de façon de plus en plus décloisonnée, en capacité d'engendrer innovations tout en impliquant un maximum l'ensemble des parties prenantes ;

- un nouvel état d'esprit entrepreneurial, nécessaire dans le renouvellement de paradigme exigé par la conjoncture actuelle.

L'aboutissement : l'économie bleue de Gunter Pauli, qui propose de ne pas opposer croissance (une croissance sans limites nous dit-il) et écologie. En vertu de quoi ? Il s'agit, tout simplement, pour l'industrie de copier la nature. Cette science a un nom : le biomimétisme. L'un de ses plus fervents porte-paroles est le chercheur Idriss Aberkane, qui l'inscrit dans une économie plus globale, celle de la connaissance (ABERKANE, 2015). Le principe est sommaire : la nature est une bibliothèque qui contient une quantité d'informations astronomique et (très) largement sous-exploitée, si ce n'est trop souvent de manière destructrice. On continue ainsi le plus souvent, nous-dit-il, « de brûler cette bibliothèque au lieu de simplement l'explorer et s'en inspirer, comme on photocopierait un livre pris dans l'un de ses rayons avant de le reposer à sa place et préserver ainsi la biodiversité ». Des perspectives qui pourraient créer des millions d'emplois allant de la recherche à la production industrielle à grande échelle, en passant par la transformation des matières premières et/ou la réutilisation de déchets industriels, sans pertes !

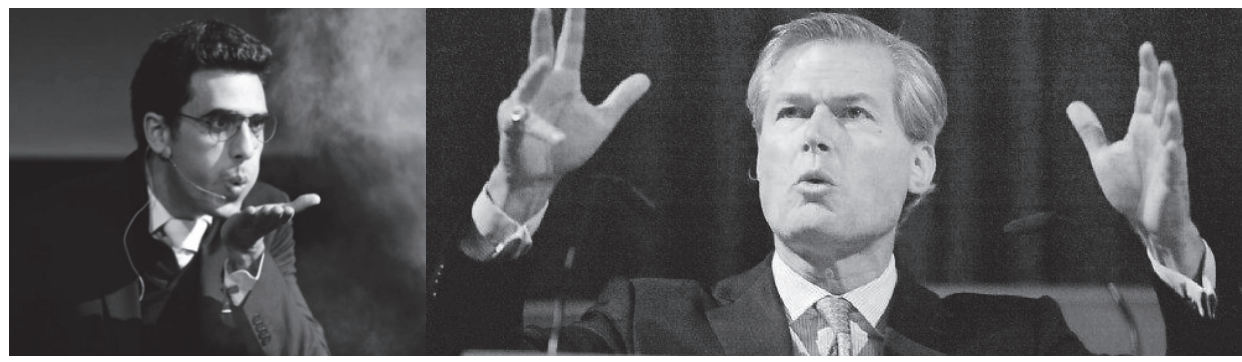

Figure 2.2.1. Idriss Aberkane (à gauche) et Gunter Pauli - (c) François Tancré, Eventpixr \& (c) Rex, Sipa/ Keith Mindham

Des usines fonctionnant sur ces modèles vertueux existent (PAULI, 2013) : en s'inspirant de la nature, il est par exemple possible de fabriquer des champignons à base de marc de café à destination des industries agroalimentaires et textiles. Ou encore, via une usine en Afrique du Sud, de transformer des pierres récupérées dans des carrières, en papier ! Plus de 200 entreprises fonctionnent sur ces principes à date, une première goutte d'eau dans l'océan de la production industrielle. 


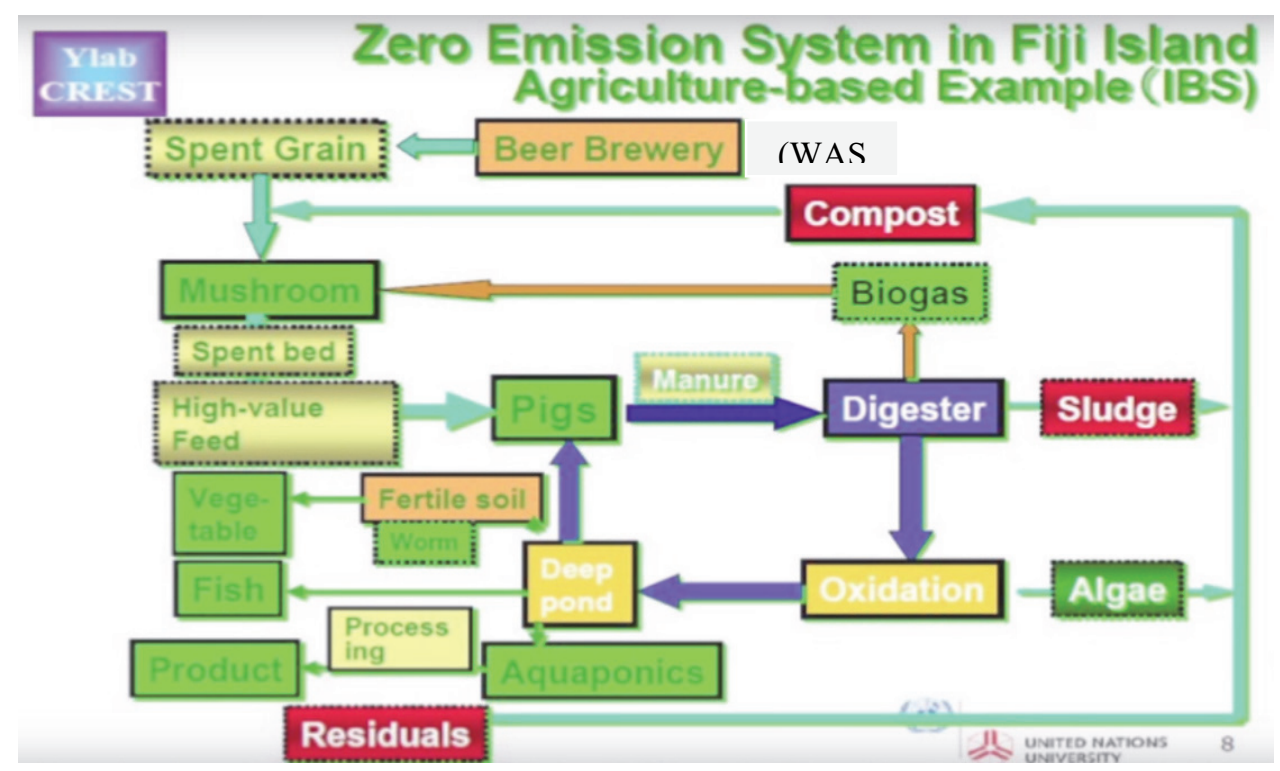

Figure 2.2.2. Traitement des déchets ZERO EMISSION dans une usine-écosystème aux Iles Fidij3 - Source : Jean Staune

Alors justement, quels impacts sur les procédures d'innovation dans les grands groupes et les consortiums industriels? Il s'agit de prendre en considération les aspects environnementaux dès la phase de conception du produit, via la notion de « récupérabilité ». " Si un déchet potentiel (par essence non récupérable) est présent dans votre produit, vous devez changer la composition de votre produit », prévient Jean Staune en citant (MCDONOUGH \& BRAUNGART, 2011) ${ }^{4}$ (ALLODI, 2013). On parle alors « d'éco-conception ».

Celle-ci ouvre la porte à une transformation du modèle économique actuel, créant une passerelle vers une économie dite de la fonctionnalité, au sein de laquelle il ne sera bientôt plus nécessaire de posséder un objet, mais simplement de l'emprunter pour une durée d'usage déterminée. Une fois ce temps écoulé, l'objet est alors récupéré par le producteur qui prend à sa charge son recyclage / reconditionnement, à $100 \%$.

Changer les règles du jeu...

...c'est aussi et surtout, replacer l'humain au cœur de l'ensemble des considérations économiques, sociétales, d'innovation. L'humain c'est donc l'opérateur, c'est le manager (et le coach), le leader ou le chef d'entreprise, c'est celui qui consomme également, celui qui participe à l'enrichissement d'un écosystème (familial, sociétal), et qui a un impact (et donc une responsabilité) de plus en plus global au travers des moyens qui lui sont conférés. Et ce notamment au cœur même des entreprises. Il s'agit de mettre l'économie au service de l'Homme, et non l'inverse. Il s'agit d'innover par et pour l'Homme, qu'il soit proche (famille, amis) ou plus lointain (à l'échelle d'un pays, d'un continent ou du globe). Et cela commence par soi. Par les « Soi », contextualisés, dans les sphères personnelles, mais aussi professionnelles et publiques. Comme Emmanuel Faber, PDG de Danone le mentionne si bien dans un entretien avec Sœur Cécile Renouard (ENTRETIENS-DE-VALPRE, 2014), il n'existe pas plusieurs éthiques : l'éthique de l'entreprise est la même que l'Ethique avec un grand $\mathrm{E}$ dès lors que des opérateurs humains donnent vie à un système de production.

3. Les déchets produits par une brasserie sont mélangés avec 3 espèces de champignons différents ce qui les rend comestible (pour des cochons). Les excréments produits sont injectés dans un digesteur produisant du biogaz, mais aussi des algues vertes (pouvant être nocives pour l'environnement). Or, les algues nourrissent sept espèces de poissons (et une espèce d'écrevisse) se relayant pour les digérer. 
Depuis quelques années déjà, de nouveaux moyens de management ou de conduite de projets sont à puiser dans la vague émergente d'outils qui promeuvent une conscience individuelle et collective. Des entreprises libérées à la holacracie (système de gouvernance basé sur l'intelligence collective), en passant par le bonheur au travail (GUILLAUD, 2016), (SHERIDAN, 2015) et les tous nouveaux Chiefs Happiness Officers, il s'agit d'autant d'initiatives désireuses de donner à l'humain une place centrale dans les unités de production et de l'accompagner dans son développement. Parmi ces outils, l'auteur cite avec enthousiasme la Théorie U, qu'il commence à partager avec ses étudiants de l'ISEP. Développée par Otto Scharmer (MIT) à partir de 2009 (SCHARMER, 2016), sur la base d'une recherche poussée et de nombreux retours terrains, il s'agit de passer par trois étapes successives pour implémenter des idées nouvelles et qui soient profondément assujetties à un « Sens » :

- ouvrir son esprit, se montrer curieux, collaboratif, moteur dans l'analyse et le traitement des informations (étape primordiale de tout processus d'innovation);

- ouvrir son cœur, se placer dans des dispositions bienveillantes envers soi et les autres (collègues, partenaires, futurs utilisateurs / clients, environnement), intégrer au sein du processus d'innovation, pleinement et de façon tenace, une notion éthique de produit / service à destination du « bien commun »;

- ouvrir sa volonté en expérimentant et ressentant la "présence », c'est-à-dire, le sens individuel et collectif des actions menées, les conséquences de ce «qui fonctionne et qui ne fonctionne pas » issues des choix réalisés, et ce à différentes échelles, afin de placer l'Homme (le soi et les autres) au cœur d'un processus de vie harmonieux, dans la durée. Puis de favoriser le passage à l'action.

En une phrase, l'auteur la traduirait par :

\section{IMPORTANT. Think Global, Feel Personal, Do Local (but not alone) \& Share It}

« Nous sommes passés d'une économie industrielle - où l'on embauchait des bras - à une économie de la connaissance - où l'on embauchait des têtes - et maintenant à une économie humaine - où l'on embauche des cœurs. » (Dov Seidman, consultant américain)

Enfin, changer les règles du jeu...

...c'est appliquer une nouvelle donne aux questions de l'éducation et de la formation. Une éducation, elle aussi, à redynamiser tant elle semble sous perfusion depuis des années, et ce autant sur le fond que sur la forme. Via une forme de transmission et de partage notamment, en partant d'expériences vécues, ayant démontré leurs efficacités économiques, sociales et environnementales, le tout en s'appuyant sur la diversité des perceptions de la réalité d'aujourd'hui. Via une transmission des valeurs, face aux menaces de déracinement, accompagnée par des prises de positions et des actes forts, symboliques, suscitant le rêve, ouvrant une fenêtre sur l'espoir.

L'éducation a, en ce sens, un devoir d'utopie, et doit être portée par une mission en adéquation avec les besoins apparemment contradictoires de notre temps : pragmatisme et idéal, matériel et spirituel, où l'on enseigne autant de compétences techniques, fonctionnelles que du savoir-être, ancré sur soi et sur l'Autre ainsi que sur la conscience des enjeux spatio-temporels qu'il nous est donné l'opportunité d'affronter aujourd'hui et demain.

Quant à l'aspect de la formation (professionnelle notamment), il s'agit de la développer autour des valeurs précitées tout en se voulant pragmatique et tournée vers les millions de personnes qui risquent de se retrouver sans emploi, balayées par l'ère du numérique, celle de l'intelligence artificielle (TUAL, 2016) et de la robotisation. Un accompagnement et un soutien forts sont exigés, avec des moyens humains et financiers dédiés, des compétences et des connaissances aigües dans les environnements socioprofessionnels des différentes branches (mais aussi entre les branches!), où chaque individu pris en charge est valorisé, où est donné à chaque accompagnant les moyens de son action, où sont créés des ponts interdisciplinaires permettant la mise en place de systèmes de vases communicants en termes 
d'expertises et d'emplois. Et qui sont à tester dans des contextes territoriaux donnés, en tenant compte de leurs spécifications sociales, culturelles et professionnelles. Pour créer des exemples, des cas d'école reproductibles.

\subsection{Alternatives économiques : bien regarder à gauche et à droite, avant de traverser}

Ce qui semblait être considéré il y a encore quelques années comme une utopie, vient d'être proposé en référendum chez nos voisins Helvètes, début juin : un revenu de base inconditionnel. Le principe est le suivant: chaque citoyen bénéficie d'une allocation inconditionnelle quels que soient ses revenus, son statut professionnel ou son statut marital. Elle s'élève à $2250 €$ par adulte (soit $50 \%$ d'un salaire médian en Suisse) et $565 €$ par mineur. Sans surprise, le «non » l'a largement emporté (à $79 \%$ ), mais pour Daniel Häni, il s'agit simplement d'une étape de parcours et déjà un succès en soi. " Dans vingt ans, nous aurons le revenu inconditionnel de base, peut-être même plus tôt », confiait-il au quotidien suisse Le Temps (ZUND, 2016). Sachant que des initiatives expérimentales se déploiement de plus en plus, notamment en Europe du Nord, il serait sans doute opportun d'explorer cette piste de façon cohérente et pragmatique.

En effet, il pourrait s'agir là d'une initiative extrêmement impactante, et sans doute une étape-clé dans la mutation économique et a fortiori industrielle. Cette mesure pourrait contribuer à déverrouiller les pressions économiques et sociales permettant aux populations en précarité, en reconversion professionnelle, en formation, mais aussi à la jeunesse d'envisager le rapport au travail, et à la vie en général, d'une façon tout à fait différente, voire bouleversante. N'est-ce pas d'un bouleversement dont nous avons besoin aujourd'hui pour orienter l'énergie de tout un chacun dans la résolution des défis planétaires qui nous attendent, afin de proposer une société plus humaine, moins soumise aux pressions de la compétitivité et des marchés tout en favorisant l'émergence d'une intelligence collective ?

Or tout cela demande du temps. Et on le sait toutes et tous, lorsque nous travaillons pour gagner notre pain quotidien, nous avons bien souvent d'autres choses à penser qu'à sauver la planète, réfléchir au sens de notre existence et à celle de nos congénères. Pourquoi ne pas, par exemple, accompagner ces revenus « inconditionnels» d' incentives» adaptées aux différents contextes. Elles pourraient ainsi favoriser le passage à l'action, en rendant par exemple un service civique ou environnemental grâce à ce «nouveau temps » qui nous serait imparti. Non plus vis-à-vis d'un travail, mais vis-à-vis d'activités qui jalonneraient nos journées, notre vie.

Car oui, dans ce paradigme, le travail tendrait à disparaître au profit d'activités qui verraient naître des initiatives liées à la mise à disposition de nos compétences / connaissances / expertises / qualités individuelles / informations au profit de différentes entreprises / associations / structures / causes en échange de biens / services / bitcoins / troc / monnaies locales, internationales, reconnues d'utilité publique et partagées dans différents cercles économiques. Ainsi il n'y aurait plus de valeur associée à un travail, mais des valeurs associées aux impacts créés par les activités que l'on mènerait, et ce, par exemple, selon différentes sphères :

- la sphère individuelle: nous exerçons une activité pour notre bien-être personnel, pour notre stimulation intellectuelle, pour prendre soin de notre état de santé, afin de nous sentir mieux dans notre vie / pour nos proches / dans la société, pour être plus heureux, consommer mieux, participer, être actif et partager notre état de bonheur ;

- la sphère familiale : une activité collective au profit de / avec notre famille, nos enfants, parents ou grands-parents, génératrice de lien et de solidarité intergénérationnelle ;

- la sphère amicale et sociétale : une activité en support de la société dans laquelle nous vivons, un investissement dans notre quartier, notre ville, auprès des êtres qui partagent un " vivre ensemble »;

- la sphère globale : agir pour l'économie au sens de l'oikonomia (étymologiquement, « la gestion de la maison »- dans le cas présent, la planète), pour l'environnement, l'ouverture et la transmission interculturelle, pour appliquer et promouvoir l'humanisme et l'écologie. 
A chacune de ces activités serait attribuée une évaluation de son impact, sur la base des heures qu'on y aurait consacré et de l'importance qu'elle aurait revêtu dans un contexte particulier, permettant de calculer des contreparties financières et/ou sous d'autres formes.

On voit alors à quel point le « chantier » industriel est imbriqué, indissociable de celui économique (et politique), lui-même nécessitant de s'appuyer sur un socle de valeurs fondamentales de l'humanité. L'ensemble des aspects cités sont bien évidemment loin d'être exclusifs, voire exhaustifs. D'autres initiatives, peut-être même plus performantes, existent sans doute. Mais, voyez comme il est difficile, voire impossible, de décorréler l'innovation et le progrès industriels d'un socle économiquement viable, sans placer au cœur du système l'humain et ses environnements.

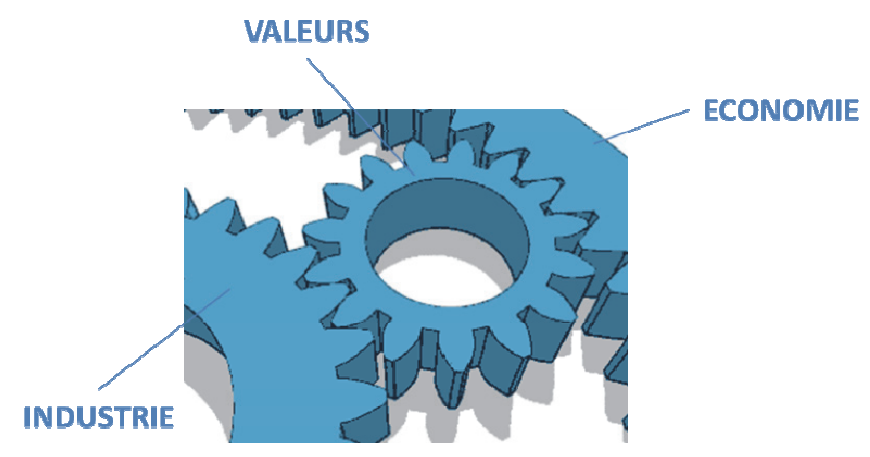

Figure 2.3. Articulation des politiques d'innovation

En conclusion, l'on pourrait se demander encore pourquoi, alors que nous avons les technologies, les compétences, les savoirs et les expertises, les méthodes et outils, que nous faisons des bilans, et, qu'a priori, nous apprenons de nos erreurs, alors même qu'un embryon de conscience planétaire semble émerger, nous restions au choix piégés, démunis, désintéressés, préoccupés par l'urgence du courtterme, non concernés, bref, étrangers (aliénés) à ces problématiques. Il existe bien sûr des facteurs bloquants, aggravants, profondément ancrés dans l'inconscient collectif et l'idée n'est pas tant là de les énumérer ou de les expliquer, que de réfuter tout simplement la question ! Car la solution n'est assurément pas là ! Et du coup, remplacer cette question par une autre : est-on en mesure de créer et d'accompagner une nouvelle génération d'explorateurs, entrepreneurs, inventeurs, innovateurs, influenceurs en capacité d'intégrer de façon profonde et durable ces enjeux, d'expérimenter à grande échelle et ainsi valider les preuves de concepts en vue d'entraîner dans leur sillage les populations déracinées?

\section{Corollaires : impacts sur l'innovation}

\subsection{Cultures}

L'idée est ici de favoriser l'émergence non pas d'une mais de différentes cultures d'innovation, adaptées et optimisées en fonction de chaque contexte (entreprise - start-up, PME, Multinationale, industrie, modèle économie, système politique) avec la nécessaire exigence d'être liées à un point d'ancrage inamovible : l'humain, partout et à tout moment. Toute innovation devra ainsi justifier sa raison d'être par sa capacité à se construire avec, autour et pour l'Homme, aujourd'hui et demain. Comptetenu des enjeux actuels, favoriser les initiatives permettant de « rembourser » (combinaison de « quickwins » et d'actions stratégiques sur le long-terme) les dettes économiques et écologiques que nous avons mises en places, au lieu, simplement, d'éviter de « trop » les aggraver.

\subsection{Visions et stratégies}

Ces cultures doivent être incarnées, portées par des décideurs conscients des enjeux et de leurs responsabilités, en capacité de bousculer les clivages en place et de substituer les logiques de compétitivité et de profit à outrance (et dans lesquelles l'enjeu humain n'est adressé que de façon partielle), par celles de 
créativité, co-réalisation, partage (des idées, des connaissances et des richesses engendrées) via des implications pluridisciplinaires et transgénérationnelles. L'innovation est ici représentée par un changement d'état d'esprit et par l'aboutissement au travers d'expérimentations d'un processus Ressenti $\rightarrow$ Conscience $\rightarrow$ Verbalisation $\rightarrow$ Décision $\rightarrow$ Action arrivé à (une certaine) maturité, enclin à se remettre en question, à évoluer et à se perfectionner de façon agile et continue.

\subsection{Processus}

S'appuyer sur des mécanismes pour la plupart déjà existants dans différentes sphères (et dont certains ont été évoqués précédemment), qu'il s'agit d'adapter aux contraintes de ses propres organisations (individuelle, familiale, entreprise, société, monde). Car l'innovation réside de plus en plus dans la capacité à trier, relier et analyser les informations à notre disposition en vue de les sélectionner et de les combiner de la manière la plus optimale possible pour, enfin, prendre le risque de les transposer à ses propres environnements. Et de trouver les arguments pour convaincre ses partenaires de l'utilité à le faire!

\subsection{Outils}

Enfin, il s'agit de promouvoir et d'utiliser les outils avec le souci de démontrer la faisabilité des innovations engagées. Là encore, ces outils existent, qu'ils soient managériaux ou technologiques, dans leur majeure partie. Il est de notre devoir de nous donner les moyens de nos ambitions en les détectant, les apprivoisant et en leur donnant un usage en accord avec les culture, vision / stratégie et processus énoncés.

\section{Synthèse}

Après avoir, dans une première partie, analysé les effets (majoritairement néfastes) des politiques industrielles sur trois composantes clé de l'évolution humaine que sont l'économie, la société et l'environnement, nous avons présenté trois axes de réflexion (technologique, des valeurs sociales et sociétales, des alternatives économiques) devant contribuer à une mutation de la démarche industrielle, en capacité de provoquer les sursauts nécessaires à l'envisagement d'un futur. S'attaquer à la problématique d'une pérennisation de l'industrie ne pourrait aboutir dans le cas où l'un de ces axes serait laissé de côté. Il apparait donc fondamental d'enclancher d'ores et déjà cette évolution tri-partite.

Et ce grâce à l'innovation, motrice de tout processus évolutif. Toutefois, le cas particulier de l'industrie met en lumière, au regard de l'urgence de la situation, la nécessité d'agir à différents degrés et de façon ouverte et coordonnée, avec un prérequis non négociable : modifier les fondements même des cultures et paradigmes inhérents à l'innovation en replaçant l'Homme au centre des enjeux. En deux mots : repenser en profondeur les modes de production ainsi que l'Utilité des biens et des services proposés.

Disposant d'ores et déjà d'un panel impressionnant d'outils, moyens, mécanismes issus des intelligences collectives humaines ou naturelles, le principal défi de l'innovation réside dans la mise en pratique de ces potentielles solutions et de leur acceptation à grande échelle par les décideurs (largement majoritaires) issus du «monde postmoderne ».

De l'intelligence à faire émerger de nouvelles pratiques entrepreneuriales, industrielles, économiques sur un laps de temps extrêmement réduit dépendent les contours sociaux, économiques et environnementaux que prendra le monde de demain, et ce à un degré encore jamais égalé dans l'histoire.

\section{Les références / la bibliographie}

ABERKANE I., (2015, 02 25). Consulté le 09 05, 2016, sur http://www.dailymotion.com/video/x2joni8.

ADEME., (2016). Consulté le 09 05, 2016, sur http://www.ademe.fr/.

ALLODI E., (2013, 03 11). Consulté le 09 05, 2016, sur https://www.youtube.com/watch?v=mvznik6tG4E. 
BANQUE MONDIALE, (2016). Consulté le 09 05, 2016, sur http://donnees.banquemondiale.org/indicateur/NV.IND.TOTL. ZS?end $=2014 \&$ start $=1995 \&$ view $=$ chart.

BAUMARD P., (2012). Le vide stratégique. Paris: Editions CNRS.

BAumard P., (2014, 03 13). Consulté le 09 05, 2016, sur Xerfi Canal: https/www.youtube.com/watch?v=9laVBd5iDgU.

BPI-FRANCE, (2016). Consulté le 09 05, 2016, sur http://big.bpifrance.fr/.

BuSSARD S., (2016, 01 15). Consulté le 09 05, 2016, sur LE-MONDE.fr: http://www.lemonde.fr/planete/article/2016/01/15/ dans-1-oklahoma-le-gaz-de-schiste-provoque-des-seismes-a-repetition_4847904_3244.html.

CEBIT, (2016). Consulté le 09 05, 2016, sur www.cebit.de/home.

DENIS J.-P., (2014, 11 6). Consulté le 09 05, 2016, sur Xerfi Canal: https://www.youtube.com/watch?v=A339gxxkccU.

ENTRETIENS-DE-VALPRE. (2014, 11 14). Consulté le 09 05, 2016, sur https://www.youtube.com/watch?v=hSV4yvMdIZ0 $\&$ feature $=$ youtu.be $\& \mathrm{t}=21 \mathrm{~m} 33 \mathrm{~s}$.

FOUCART S., (2014, 12 10). Consulté le 09 05, 2016, sur http://www.lemonde.fr/medecine/article/2014/12/10/la-pollutionmet-en-danger-le-cerveau_4538177_1650718.html.

GADREY J., (2014). Consulté le 09 05, 2016, sur http://alternatives-economiques.fr/blogs/gadrey/2014/03/10/210-milliards$\mathrm{d} \% \mathrm{E} 2 \% 80 \% 99$ euros-de-dividendes-oui-mais $\% \mathrm{E} 2 \% 80 \% \mathrm{~A} 6 /$.

GIEC, (2014). Changements climatiques. https://ipcc.ch/pdf/assessment-report/ar5/syr/AR5_SYR_FINAL_SPM_fr.pdf.

GOUVERNEMENT, (2014). Consulté le 09 05, 2016, sur http://www.economie.gouv.fr/files/files/PDF/nouvelle-france-industrielle-sept-2014.pdf.

GOUVERNEMENT, (2016). Consulté le 09 05, 2016, sur http://www.gouvernement.fr/partage/3813-1-industrie-en-france.

GuILLAUD H., (2016, 06 11). Consulté le 09 05, 2016, sur LE-MONDE.fr.

INSEE, (2016). Consulté le 09 05, 2016, sur http://www.insee.fr/fr/themes/info-rapide.asp?id=40.

KAIMAN J., (2014, 03 20). Consulté le 09 05, 2016, sur theguardian: https://www.theguardian.com/sustainable-business/ rare-earth-mining-china-social-environmental-costs.

LALOUX F., (2014). Reinventing Organizations. Bruxelles: Editions NELSON PARKER.

LATRIBUNE.fr, (2016, 01 19). Consulté le 09 05, 2016, sur La Tribune: http://www.latribune.fr/economie/international/ la-4e-revolution-industrielle-menace-plus-de-5-millions-d-emplois-543777.html.

LE-MONDE.fr, (2016, 05 26). Consulté le 09 05, 2016, sur http:/www.lemonde.fr/sante/article/2016/05/26/plus-de-500000-morts-par-cancer-dans-le-monde-associees-a-la-crise-de-2008_4926524_1651302.html.

MATTEI J.-F., (2015). L’homme dévasté. Editions GRASSET.

MCDONOUGH W., \& BRAUNGART, M. (2011). Cradle to Cradle. Editions MANIFESTÔ.

MORIN E., (2011). La Voie. Editions FAYARD.

OPEO, (2014). Consulté le 09 05, 2016, sur http://www.opeo-conseil.fr/.

OPEO, (2016). Consulté le 09 05, 2016, sur http://www.challenges.fr/tribunes/20160719.CHA1969/quelle-sera-la-franceindustrielle-de-2025.html.

OXFAM, (2015). Insatiable richesse, toujours plus pour ceux qui ont déjà tout. http://www.oxfamfrance.org/sites/default/files/ file_attachments/rapport_oxfam_insatiable_richesse_0.pdf.

PARASOTE V., (2013, 03 15). Consulté le 09 05, 2016, sur http://twi-terre.net/index.php/infos-sourcees/55-batterie-lithiumion-un-poids-plume-aux-lourds-impacts.

PATRIUM, (2016). Consulté le 09 05, 2016, sur http://www.patrium.fr/.

PAULI G., (2013, 06 06). Consulté le 09 05, 2016, sur TEDx: http://www.dailymotion.com/video/x2joni8.

PAULI G., (2015). Consulté le 09 05, 2016, sur http://www.theblueeconomy.org/.

RIES E., (2011). Lean Startup. Editions PEARSON.

SALMON K., (2015). Consulté le 09 05, 2016, sur http://www.kurtsalmon.com/uploads/2015_POV_\%20U\%26I_Indutrie\%204.0 -WEB_VF.pdf.

SAVE., (2016). Consulté le 09 05, 2016, sur https://www.save.co/fr/fr. 
SCHARMER O., (2016). Theory U - Leading from the Future as it Emerges (éd. 2e). Berret-Koeler Publishers.

SHERIDAN R., (2015). Joy, Inc. Editions PENGUIN.

SUPIOT A., (2015). La gouvernance par les nombres. Editions FAYARD.

STAUNE J., (2015). Les clés du Futur. Paris: Editions PLON.

STAUNE J., (2016). Consulté le 09 05, 2016, sur https:/www.youtube.com/watch?v=NZKqPoQiaDE\&feature=youtu.be\&t= $14 \mathrm{~m} 2 \mathrm{~s}$.

TESLA, (2016). Consulté le 09 06, 2016, sur https://www.tesla.com/fr_FR/gigafactory.

TUAL M., (2016, 06 17). Consulté le 09 05, 2016, sur LE-MONDE.fr: http://www.lemonde.fr/pixels/article/2016/06/17/ intelligence-artificielle-google-lance-un-groupe-de-recherche-europeen-sur-l-apprentissage_4952666_4408996.html.

ZUND C., (2016, 03 18). Consulté le 09 05, 2016, sur LE-TEMPS: https://www.letemps.ch/suisse/2016/03/18/entrepreneurlance-initiative-un-revenu-base-inconditionnel. 